\title{
An Animal Model for Trigeminal Neuralgia by Compression of the Trigeminal Nerve Root
}

Dao-Shu Luo, MD1, Ting Zhang, MD², Chang-Xu Zou, MD², Zhong-Fu Zuo, MD², Hui Li, MD², Sheng-Xi Wu, MD, PhD², Wei Wang, MD'1, and Yun-Qing Li, MD, PhD ${ }^{1,2}$

From: ${ }^{1}$ Department of Anatomy, Histology and Embryology, Basic Medical College, Fujian Medical University, Fuzhou, Fujian, China; ${ }^{2}$ Department of Anatomy, Histology and Embryology, K.K. Leung Brain

Research Centre, The Fourth Military Medical University, Xi'an, Shaanxi,

China

Dr Luo, Dr. Wang and Dr. Yun-Qing Li are with the Department of Anatomy,

Histology and Embryology, Basic

Medical College, Fujian Medica

University, No. 88 Jiaotong Road

Fuzhou, Fujian Province, 350004, PR

China

Dr. Zhang, Dr. Zuo, Dr. Hui Li and Dr. Yun-Qing Li are with the Department of Anatomy, Histology and Embryology, K.K. Leung Brain Research Centre, The Fourth Military

Medical University, No. 169 West Changle Road, Xi'an, Shaanxi Province, 710032, PR China.

Address correspondence: Yun-Qing Li, MD

Department of Anatomy, Histology and Embryology

K.K. Leung Brain Research Centre The Fourth Military Medical University, Xi'an 710032, China.

E-mail: deptanat@fmmu.edu.cn and Wei Wang, MD

Department of Anatomy, Histology and Embryology,Basic Medical College,

Fujian Medical University,Fuzhou, 350004, China.

E-mail: wangweio976@163.com

Disclaimer: This work was supported by grants from the National Natural Science Foundation of China (Nos:30771133, 30971123 and 31010103909)

Conflict of interest: None.

Manuscript received: 10/16/2011

Revised manuscript received: $12 / 10 / 2011$

Accepted for publication: 12/27/2011

Free full manuscript: www.painphysicianjournal.com
Background: Microvascular compression of the trigeminal nerve root is a major cause of most trigeminal neuralgia (TN) in patients; however, no reliable animal model to further study the pathogenesis of TN currently exists.

Objective: Our objective was to establish a novel and practical animal model for TN by chronic compression of the trigeminal (CCT) nerve root in rats, which would provide a better animal model to mimic the clinical feature of TN on the research of the pathogenesis of TN.

Study Design: A randomized, double blind, controlled animal trial.

Methods: Sixteen adult male Sprague-Dawley rats (200-220 g) were randomly divided into 2 groups: one group that received chronic compression of the trigeminal nerve root (the CCT group, $n=8$ ) and another group that received sham operation without compression (the sham operation group, $n=8$ ). A small plastic filament was retrogressively inserted into the intracalvarium from the inferior orbital fissure until it reached the trigeminal nerve root for compression in CCT group. Animal behaviors were observed for 4 weeks after operation. Immunohistochemistry of glial fibrillary acidic protein (GFAP), isolectin B4 (IB4), substance $\mathrm{P}$ (SP) and calcitonin gene-related peptide (CGRP) were performed in the trigeminal root entry zone (TREZ) and medullary dorsal horn (MDH).

Results: The orofacial mechanical allodynia and heat hyperalgesia in the CCT rats were obviously increased after the operation and lasted for 28 days. Increased facegrooming behavior was also observed in the CCT rats and continued for over 21 days, returning to baseline by day 28. Immunohistochemistry for GFAP in the TREZ revealed a progressive extension of astrocytic processes in the ipsilateral TREZ of rats in the CCT group. Furthermore, the IB4 positive immunoreactive nonpeptidergic $\mathrm{C}$-fiber terminals in the MDH were reduced for 4 weeks after the operation. Both SP and CGRP, expressed in the peptidergic C-fiber terminals, were found to be decreased in the ipsilateral MDH of CCT animals after the trigeminal nerve root injury.

Limitations: CCT animal model with a plastic filament only imitated the mechanical compression of the trigeminal root but not to display the complex vascular physiological feature as the microvascular in the TN patient.

Conclusions: The chronic compression of the trigeminal nerve root in rats effectively induced persistent orofacial neuropathic pain behaviors, and it would provide a novel and practical animal model for future research on the pathogenesis of TN.

Key words: trigeminal neuralgia, nerve root compression, animal model, mechanical allodynia, heat hyperalgesia, substance $\mathrm{P}$, calcitonin gene-related peptide, isolectin B4

Pain Physician 2012; 15:187-196 
T rigeminal neuralgia (TN) is a chronic orofacial pain syndrome characterized by paroxysmal, shock-like pain that is localized to the divisions of one or more branches of the trigeminal nerve. The pathogenesis of TN is still unclear (1-3). Thus, establishing a reliable animal model that resembles clinical TN is crucial for TN pain research.

Previous animal models of TN, such as infraorbital nerve chronic constriction injury (IoN-CCI) (4) and partial infraorbital nerve ligation (ploNL) (5), are commonly used in current researches. However, these models also have several limitations:most of them were adapted from the spinal nerve $\mathrm{CCl}$ model, the trigeminal system doses have certain unique features different from that of the spinal system (6); Most importantly, none of the TN patients had ever suffered from such similar infraorbital nerve loose ligation or partial ligation injury in clinical situations as the models showed.

Accumulated clinical evidence has shown that vascular compression of the trigeminal nerve root by an aberrant loop of artery or vein accounts for most cases of TN (7-9). Microvascular decompression of the trigeminal nerve has become an accepted and effective means of treating patients with TN in recent years; the quick pain relief of TN following such surgery also supports this etiology (10). Therefore, the aim of our research was mainly to produce a novel animal model for TN by chronic compression of the trigeminal nerve root in rats.

\section{Methods}

\section{Animals}

Adult male Sprague-Dawley rats (200 - $220 \mathrm{~g}$ ) were randomly divided into 2 groups:one group received chronic compression of the trigeminal nerve root $(n=8)$ and another group received sham operation $(n=8)$. Water and food were available ad libitum. All experimental procedures were approved by the Animal Care and Use Committee for Research and Education of the Fourth Military Medical University (Xi'an, China). All efforts were made to minimize the animal suffering and to reduce the number of animals used.

\section{Surgical Procedures}

Rats were anesthetized with pentobarbital $(40 \mathrm{mg} /$ $\mathrm{kg}$, i.p.). The surgery was performed on the right sides of the experimental animals under direct visual control using a Zeiss surgical microscope (Carl Zeiss, Inc., Jena, Germany). The rats were taped to a sterilized cork board, and the skin above the eye was shaved. Lubricating ophthalmic ointment was applied to the eyes to prevent drying damage. A small curve anterior-posterior skin incision was made above the right eye (Fig. 1A). The fascia and muscle were then gently teased laterally from the bone to retract the contents of the orbit laterally, as previously described (11). Once the orbital contents were gently deflected, the right infraorbital nerve could be seen lying on the infraorbital groove of the maxillary bone deep within the orbit. The superior surface of the loN was then separated. A small round plastic filament (diameter $0.1 \mathrm{~cm}$ ) was gently placed above the superior surface of the IoN and then inserted into the intracalvarium through the inferior orbital fissure following the proximal direction of the loN (Fig. 1B). The filament was then slowly inserted into the canal between the cerebral dura mater and the pars petrosa ossis temporalis and crossed the Meckel's cave in turns to reach the trigeminal nerve root, in accordance with the pathway of the trigeminal nerve went through in the intracalvarium. In a pilot experiment, we had measured that the distance from the inferior orbital fissure to the proximate junction of the trigeminal nerve root entering the pons was about $1.5 \mathrm{~cm}$ in adult rats. Thus, we slowly inserted the filament about $1.2 \mathrm{~cm}$ into the intracalvarium to compress the trigeminal nerve root so as to avoid contacting and injuring the pons (Fig. 1C). For the sham operation group, the right loN was exposed and left intact, and no filament was inserted into the inferior orbital fissure. The incision was closed using silk sutures (5-0).

\section{Mechanical Allodynia}

The mechanical testing procedure was modified from previously described methods (4). Rats were habituated to the testing for 3 days before baseline testing, and then were placed in a small handmade porous metal mesh cage (Fig. 1D) to habituate for 30 minutes before threshold testing. A logarithmic series of calibrated Semmes-Weinstein monofilaments (von Frey hairs; Stoelting, Kiel, WI, USA) were applied to test mechanical stimulation in the vibrissa pad of the animals to determine the stimulus intensity threshold stiffness to elicit a brisk head withdrawal response. Log stiffness of the hairs is defined as log10 (milligrams $\times 10$ ) (12). The filaments had the following log-stiffness values (the value in milligrams is given in parentheses):2.36 (23 mg), 2.44 (28 mg), 2.83 (68 mg), 3.22 (166 mg), 3.61 (407 mg), 3.84 (692 mg), 4.08 (1202 mg), 4.17 (1479 mg), 4.31 (2041 mg), 4.56 (3630 mg), 4.74 (5495 mg), 4.93 


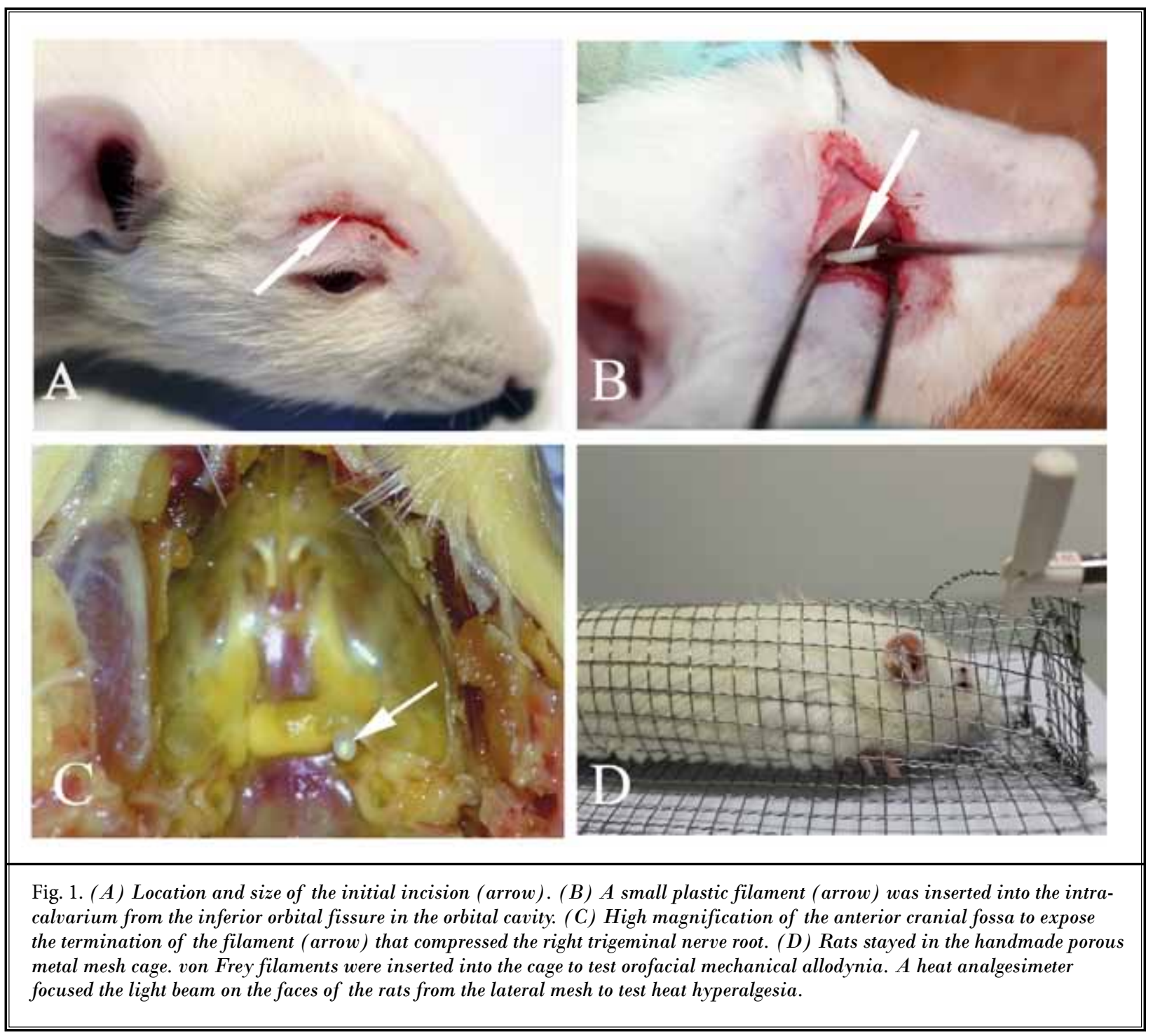

(8511 mg), and 5.07 (11749 $\mathrm{mg})$. The range of monofilaments used in this study ( $23-11749 \mathrm{mg}$ ) has been shown previously to produce a logarithmically graded slope when interpolating a $50 \%$ response threshold of stimulus intensity [calculated as log10 (milligrams $\times 10$ )] (13). Stimulations were administered when the rat was in a sniffing/no locomotion position. Each von Frey filament was applied 5 times, and a new stimulus was applied only when the rat resumed this position at least 30 seconds after the preceding stimulation. The stimulation always began with the filament that produced the lowest force and stopped when the threshold was found within the vibrissa pad. Unresponsive rats received a maximal von Frey filament force of $10 \mathrm{~g}$. The behavioral responses were used to calculate the $50 \%$ head withdrawal threshold (absolute threshold), by fitting a Gaussian integral psychometric function using a maximum-likelihood fitting method, as described in detail previously (12).

\section{Heat Hyperalgesia}

An RTY-3 radiant heat analgesimeter (Xi'an, P.R. China) was used to measure heat withdrawal sensitivity on the face in our study. Rats were loosely restrained in the same handmade porous metal mesh cage (Fig. 1D) and allowed to acclimate for 20 minutes. A highintensity light beam was focused on the IoN territory near the center of the vibrissal pad on the hairy skin 
surrounding the mystacial vibrissae to serve as a noxious heat stimulus (14). The radiant heat stimulus was a focused $5 \times 5 \mathrm{~mm}$ beam of light with a high intensity projector halogen lamp bulb (100 W) by RTY-3 radiant heat analgesimeter. Head withdrawal latencies were measured with an automatic timer to the nearest 0.01 seconds. The distance between the projector lamp bulb and the mystacial vibrissae was adjusted to be almost within one $\mathrm{cm}$. The heat nociceptive threshold was measured by determining changes in head withdrawal latency. Only rapid head withdrawal movements from the heat stimulus (with or without face grooming) were considered to be withdrawal responses. Each facial IoN territory was measured 3 times at 5-minute intervals, and the averaged values for each test were used to compute heat hyperalgesia.

\section{Face-Grooming Actions}

To assess the spontaneous behaviors of the rats in the experiment, the face grooming episodes (ipsilateral, contralateral, or bilateral face grooming) of the rats in the 2 groups were recorded by a video camera one day before and at 1, 3, 10, 14, 21, and 28 days after surgery. The video recording of face grooming was started 20 minutes after the rats adapted to the test cage. The face grooming behavior was analyzed off-line by an independent observer who was blind to the treatment of the rats. For each observation session of the rats in 10 minutes, the total time of asymmetrical face grooming episodes as unilateral strokes with the dominant paw was counted. Data were analyzed using analyses of variance (ANOVA).

\section{Immunohistochemistry}

The rats were deeply anesthetized with pentobarbital sodium (100 mg/kg, i.p.) and perfused through the ascending aorta with $200 \mathrm{~mL}$ of $0.01 \mathrm{M}$ phosphatebuffered saline (PBS, pH 7.3) followed by $500 \mathrm{~mL}$ of cold $\left(4^{\circ} \mathrm{C}\right) 4 \%$ paraformaldehyde. The brainstem and trigeminal nerve roots on both sides were dissected, post-fixed for 2 hours, and cryoprotected with a solution of $30 \%$ sucrose $(\mathrm{w} / \mathrm{v})$ in $0.1 \mathrm{M} \mathrm{PB}$ at $4^{\circ} \mathrm{C}$ for 24 hours. Transverse sections of the medulla ( $30 \mu \mathrm{m}$ thick) and longitudinal trigeminal nerve root sections $(18 \mu \mathrm{m}$ thick) were cut with a cryostat (Leica CM1800; Heidelberg, Germany) at $-20^{\circ} \mathrm{C}$. Immunohistochemistry was done according to standard protocols using primary antibodies:mouse anti-GFAP (1:5000; Chemicon, Temecula, CA, USA), rabbit anti-SP (1:100; Chemicon/Millipore, Billerica, MA, USA), goat anti-CGRP (1:1000; Chemicon/Millipore, Bil- lerica, MA, USA), and FITC-conjugated IB4 (a marker for unmyelinated C-fibers, 1:200; Sigma, St. Louis, MO, USA). Images were obtained using a confocal laser microscope (FV1000; Olympus, Tokyo, Japan). For semiquantification, the fluorescent brightness values of IB4immunoreactive (IR), SP-IR and CGRP-IR were detected on the same areas of the MDH under an IX-70 confocal microscope by using software. After the images were captured, the optical densities of the same areas of the ipsilateral superficial MDH (laminae I and II) were calculated and averaged across the 5 sections of the medulla.

\section{Statistical Analysis}

All results were expressed as the mean \pm SEM. Data from the von Frey test were analyzed as the interpolated $50 \%$ threshold (absolute threshold) in log base 10 of the stimulus intensity (monofilament stiffness in milligrams $\times 10$ ) as described previously (15). Repeated measures ANOVA (with Bonferroni confidence interval adjustment) were used for analysis. All statistical analyses were performed using SPSS Statistics 17.0 software (SPSS Inc., Chicago, IL, USA). $P<0.05$ was considered statistically significant in this study.

\section{Results}

\section{Mechanical Allodynia}

Most CCT group rats were insensitive to mechanical stimulation on the ipsilateral vibrissa pad of the nerve injury (Fig. 2A) one day after surgery, but they showed significant mechanical allodynia from day 3 through day 28 after surgery compared with baseline and the sham operation group $(P<0.05)$. However, animals in the sham-operated group displayed no obvious changes in their responses to mechanical stimuli after surgery compared with baseline $(P>0.05)$.

\section{Heat Hyperalgesia}

Before surgery (baseline), the latencies of orofacial withdrawal to noxious heat were measured for all of the animals. There was no significant difference in the latencies of orofacial withdrawal between the CCT and sham operation groups based on the test results $(P>$ 0.05 ), although during the early phase (from day one to 14 days after surgery), the sham operation group had lower head withdrawal latencies than the CCT group, and inversely the CCT group had lower head withdrawal latencies than the sham operation group during the late phase (from 14 days to 28 days after surgery). However, compared with the baseline values, both the CCT 

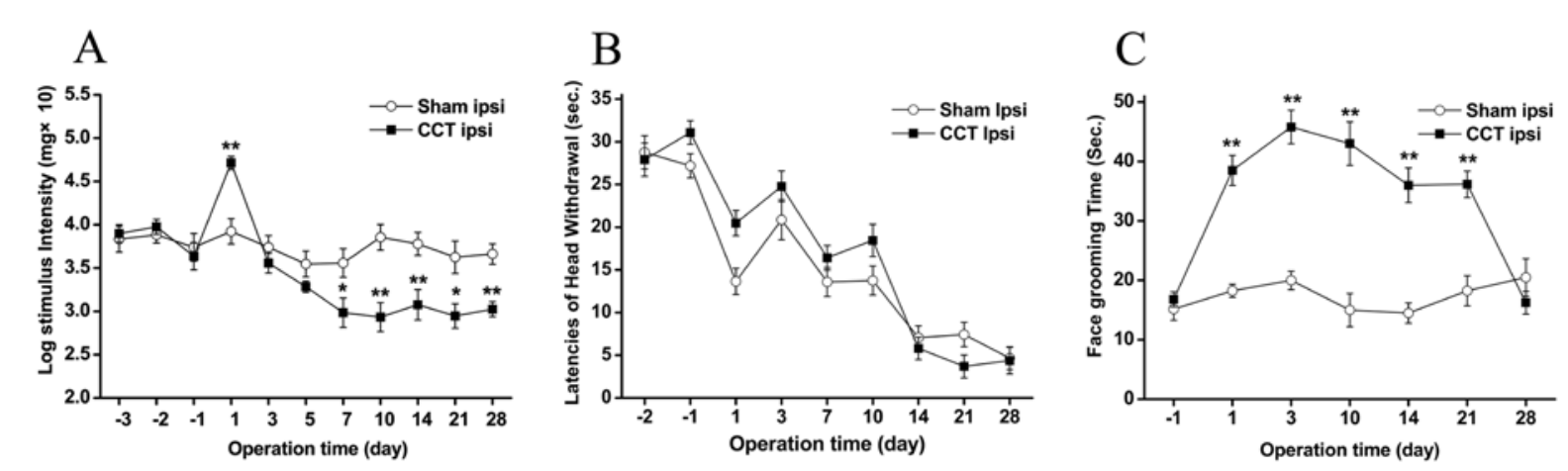

Fig. 2. Behavioral testing in the sham operation $(n=8)$ and CCT $(n=8)$ groups rats before the surgery (baseline) and after the surgery. A) The mechanical stimulation threshold responses to von Frey filaments. Although day one after surgery showed insensitivity to mechanical stimulation, compared with the sham operation group, the CCT rats showed significant mechanical allodynia after the operation that continued for at least 28 days $(P<0.05)$. B) Rats in both groups had significant decreases in the withdrawal latencies to heat stimulation after surgery compared with the baseline values $(P<0.05)$. But there was no significant difference between the CCT and sham operation groups $(P>0.05)$. C) Compared with the sham-operated rats, there was a significant increase in spontaneous face grooming behavior in the CCT rats after the surgery that lasted for over 3 weeks $(P<0.01)$; face grooming then returned to baseline by 28 days after surgery. Data are presented as the mean $\pm S E M .{ }^{*} P<0.05$; ** $P<0.01$

and the sham operation animals showed significant decreases in the latencies of withdrawal to heat stimulation after surgery $(P<0.05)$ (Fig. 2B). These data imply that both facial trauma and trigeminal nerve injury may induce orofacial heat hyperalgesia in our experiment.

\section{Face Grooming}

We calculated the asymmetrical unilateral face grooming episode time as a spontaneous behavioral response. There was a significant increase in face grooming in the CCT rats compared with the sham operation animals on post-operation day one; this lasted for over 3 weeks after the surgery (Fig. $2 C, P<0.01$ ). Animals in the CCT group had longer face grooming episodes during the early period (from one day to 14 days after surgery) than the late period (from 14 days to 28 days after surgery); however, face grooming returned to baseline levels by 28 days after surgery. Meanwhile, the rats in the sham operation group also displayed a slight transient increase in face grooming on days one and 3 after surgery; face grooming returned to baseline by day 10 .

\section{GFAP Immunoreactivity in the Trigeminal Root Entry Zone}

Our results showed that the distal extension of glial fibrillary acidic protein (GFAP)-positive astrocytic processes formed a characteristic glial dome of central tissue into the trigeminal nerve root from the central nervous system, which made a distinct borderline between the central nervous system and the peripheral nervous system on the trigeminal root entry zone (Fig. 3). We found that distal extension of GFAP-positive astrocytic processes in the trigeminal root extended progressively from the central nervous system side of the borderline to the PNS side from days 14 to 28 in the ipsilateral trigeminal root entry zone of rats in the CCT group (Fig. 3A-D), especially on days 21 (Fig. 3C) and 28 (Fig. 3D) as the arrows showed. After surgery in the CCT group, there were robust and dense GFAP-positive astrocytic processes that extended from the boundary of the central nervous system side boundary extended to the PNS side in the ipsilateral trigeminal root entry zone. However, the GFAP-IR astrocytes in the ipsilateral trigeminal root entry zone of the sham operation group showed no obvious alteration in the boundary during the post-operation period (Fig. 3A-D).

\section{Immunohistochemistry of the Central Projections of the Nociceptive Afferents in the MDH}

The isolectin B4 (IB4) staining for non-peptidergic C-fibers was confined to the superficial lamina II of the medullary dorsal horn (MDH) (Fig. 4). The IB4 density was relatively homogeneous along the medial to lateral 

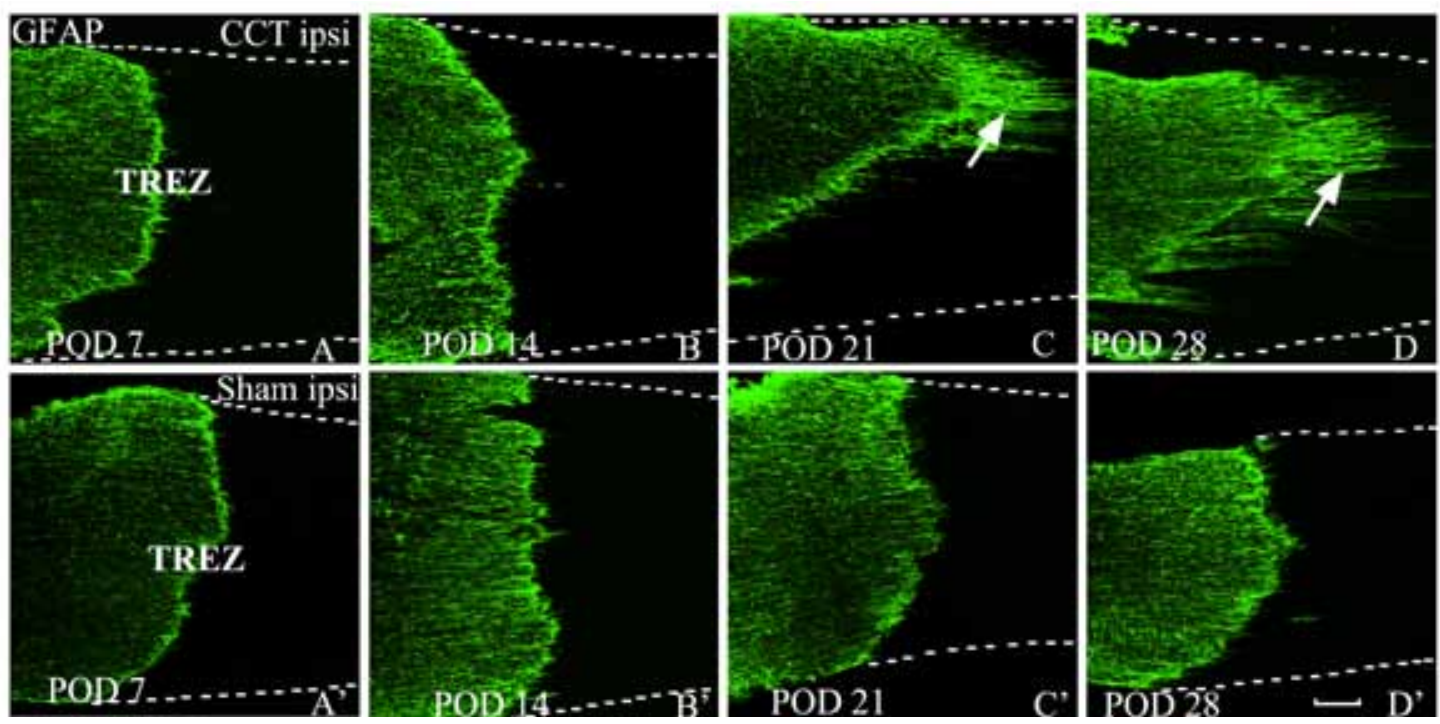

Fig. 3. GFAP immunoreactivity in the TREZ on days 7, 14,21, and 28 after surgery. The distal extension of GFAP-positive astrocytic processes formed a characteristic glial dome of central tissue into the trigeminal nerve root from the CNS, which made a distinct borderline between the CNS and the PNS on the TREZ. The GFAP-positive astrocytic processes progressively extended from days 14 to 28 after surgery in the CCT rats (A-D), especially on days 21 (Fig. 3C) and 28 (Fig. 3D) as the arrows showed. The GFAP immunoreactivity in the sham operation group showed no obvious alteration in the ipsilateral TREZ (A'$\left.D^{\prime}\right)$. Scale bar: $100 \mu \mathrm{m}$.

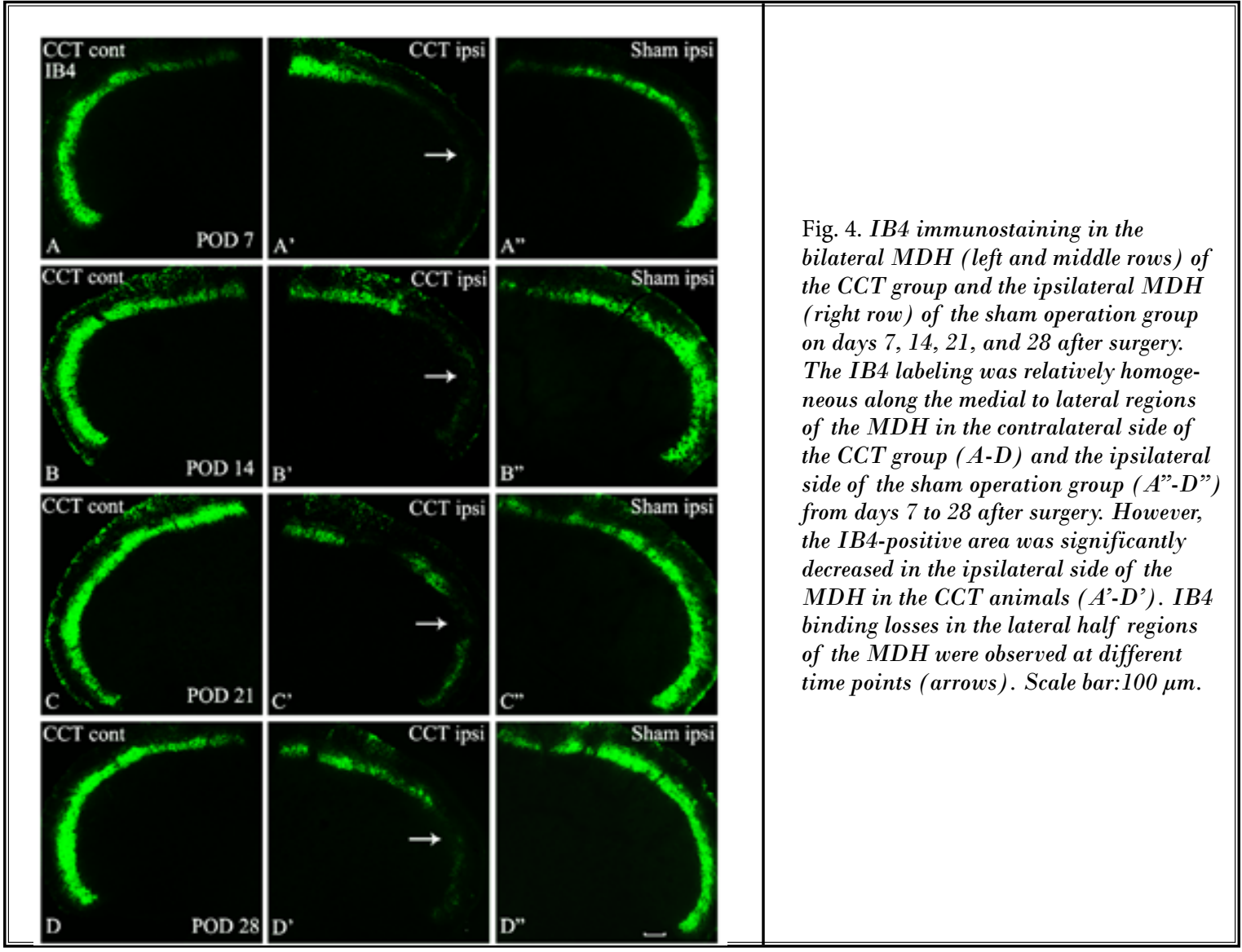



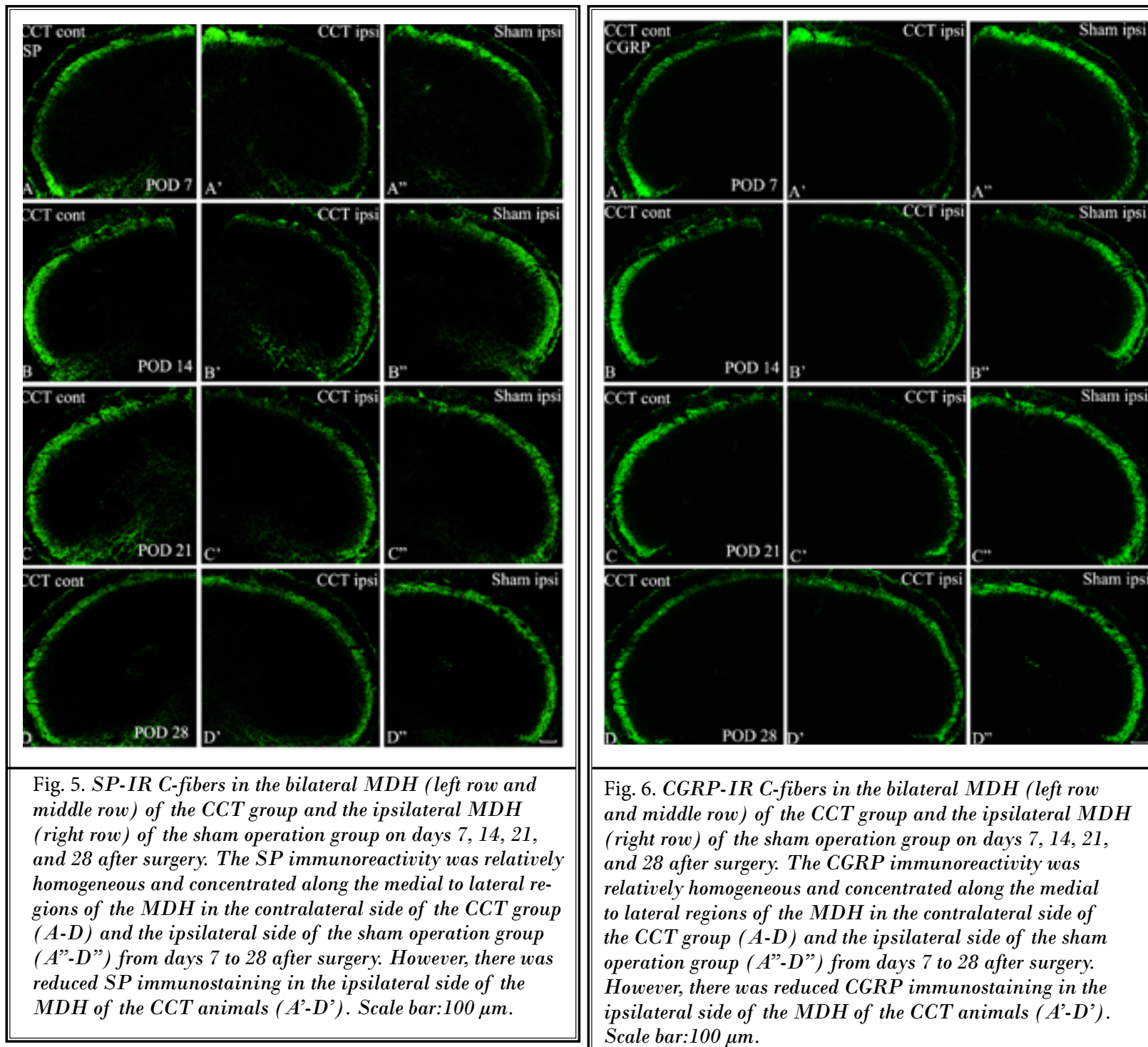

Fig. 6. CGRP-IR C-fibers in the bilateral MDH (left row and middle row) of the CCT group and the ipsilateral $M D H$ (right row) of the sham operation group on days $7,14,21$, and 28 after surgery. The CGRP immunoreactivity was relatively homogeneous and concentrated along the medial to lateral regions of the $\mathrm{MDH}$ in the contralateral side of the CCT group ( $A-D$ ) and the ipsilateral side of the sham operation group ( $A$ "- $D$ ") from days 7 to 28 after surgery. However, there was reduced CGRP immunostaining in the ipsilateral side of the MDH of the CCT animals ( $\left.A^{\prime}-D^{\prime}\right)$. Scale bar: $100 \mu \mathrm{m}$.

regions of the medullary dorsal horn in the contralateral side of the CCT group (Fig. 4A-D) and the ipsilateral side of the sham-operated group (Fig. 4A-D) from days 7 to 28 after surgery. However, in the ipsilateral side of the $\mathrm{MDH}$ in the CCT group (Fig. 4A), the IB4-positive area was significantly decreased; specifically, there was an IB4 binding loss in the lateral half of the MDH at different time points. Nociceptive peptidergic C-fibers were identified by their expression of substance $P$ (SP) and calcitonin gene- related peptide (CGRP. Both SP (Fig. 5) and CGRP (Fig. 6) immunoreactivity were observed in the lamina II of the $\mathrm{MDH}$. The values of the relative im- munodensities of IB4-IR C-fibers in the ipsilateral side of the CCT group also showed significant decreases by semi-quantification analysis (Fig. 7A, $\mathrm{P}<0.01$ ) and persisted until 28 days after the operation.

The immunofluorescence density values of SP-IR (Fig. 7B) and CGRP-IR (Fig. 7C) of C-fibers in the CCT animals showed a relatively homogeneous and modest decrease in the ipsilateral $\mathrm{MDH}$ on days $7,14,21$, and 28 after surgery $(P<0.05)$. No SP-positive area or CGRP-positive area deletion was detected in the $\mathrm{MDH}$ of either group. 


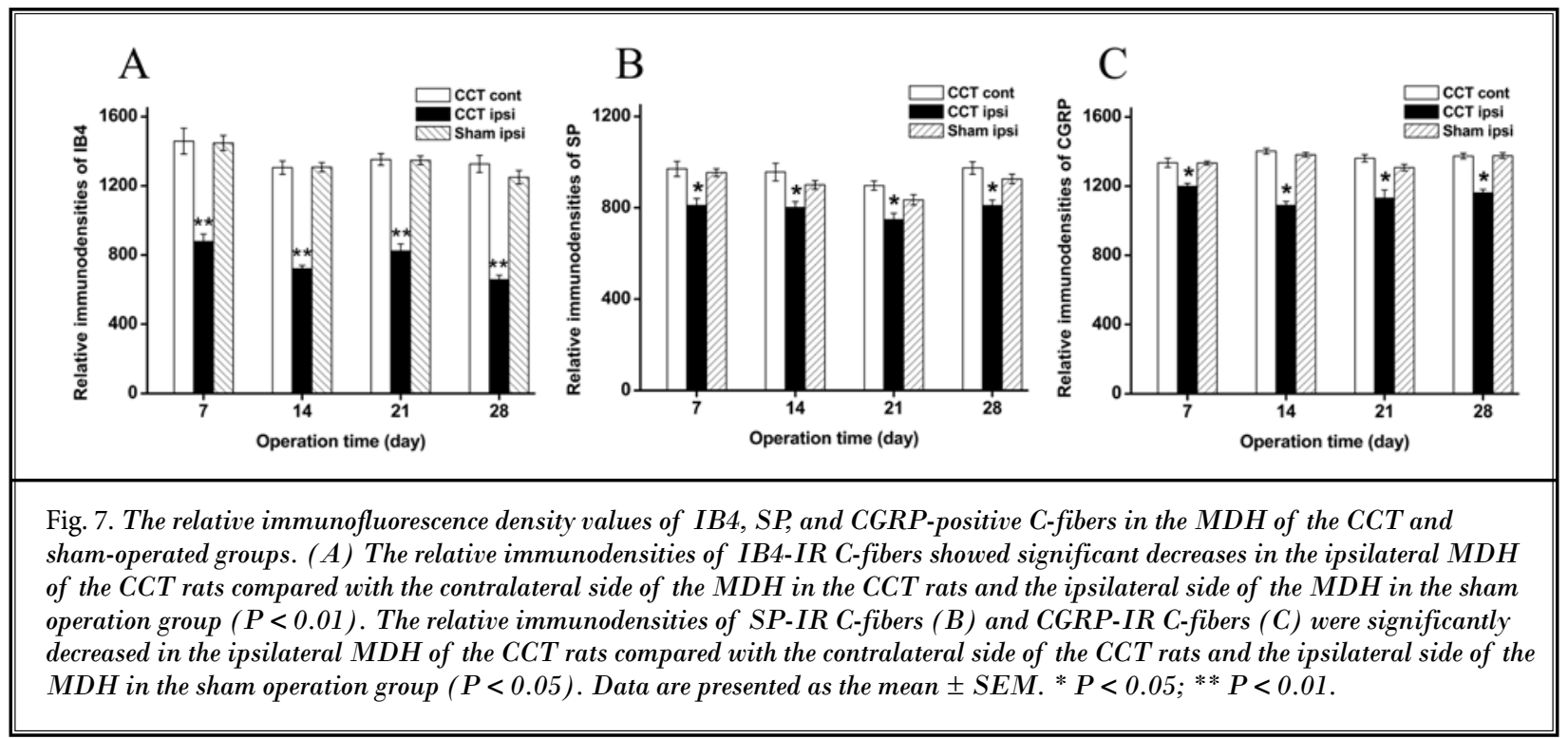

\section{Discussion}

In recent years, more and more people have been prone to believe that TN is caused by microvascular compression in the TREZ. It was found that orofacial sensory information enters the CNS via the TREZ, where sensory axons span an environment consisting of Schwann cells in the PNS and astrocytes and oligodendrocytes in the CNS (16). According to this common clinical feature in most cases of TN patients, our study established a novel animal model for TN following chronic compression of trigeminal nerve root, which would be better to mimic the clinical situation of TN. So far, an extensive analysis of responses to mechanical and thermal stimulations of the face and of spontaneous face grooming behavior observed after $\mathrm{CCl}$-loN or ploNL have been interpreted as signs of abnormal spontaneous pain-related behavior, mechanical allodynia, and heat hyperalgesia in animal models of TN $(4,5,14)$.Therefore, such behavioral tests were also performed in the CCT animal model of TN in our study.

Compared with the baseline and the sham-operated group, the CCT group had a very high mechanical threshold on the first day after the operation, indicating that the territory ipsilateral to the trigeminal nerve injury scarcely responded to the primary afferents of mechanical stimulation. This phenomenon is possibly related to the stress and trauma of surgery after trigeminal nerve root compression in rats. However, the rats in the compression group showed significant al- lodynia according to the decreased thresholds to mechanical stimuli from days 3 through 28 after surgery. These results suggest that chronic compression of the trigeminal nerve root may induce mechanical allodynia in this animal model of TN.

The withdraw latency measure was a clear expression of a pronounced heat hyperalgesia in the animal models of neuropathic pain such as CCl (17-19). Although there is not any case reports of heat hyperalgesia in TN patients from the literature, it may be that the heat hyperalgesia symptom was less severe compared with the shock-like pain when the TN was attacked which would be ignored by the patients. So we also studied the heat hyperalgesia in the CCT model of TN. Our research showed that both the compression and sham-operated animals had heat hyperalgesia. We presume that both surgical trauma and trigeminal nerve injury may induce a nociceptive heat stimulus due to nerve injury during the early phase and that significant heat hyperalgesia is a result of central sensitization by trigeminal nerve compression during the late phase. Hence, we deduced that the trigeminal nerve root compression injury also induced heat hyperalgesia in this CCT model, as previous research showed in other animal models of TN $(14,20)$.

However, face grooming behavior decreased after 3 weeks and returned to baseline at 4 weeks after the operation. Actually, although grooming behavior 
is commonly used in orofacial pain research in animals (21), it is still controversial because increased grooming behavior can serve either as a symbol for early hypesthesia after chronic constriction injury to the rat's infraorbital nerve studies (4) or represent the intense pain induced by formalin injection into the orofacial region (21). Therefore, it is possible that face grooming did not match the real pain time window that is featured by the long-lasting mechanical allodynia and heat hyperalgesia in our study. Further efforts are needed to address this question in the future.

According to the clinical pathological biopsy findings for the trigeminal nerve root from microvascular decompression surgery, much evidence strongly supports the hypothesis that microvascular compression can damage the trigeminal root fibers in patients with $\mathrm{TN}$; this damage is thought to be critical for the induction of TN. Immunoelectron microscopy for GFAP confirmed that the astrocytic processes were largely confined to the periphery of the lesion (22). It was also reported that massive injury to nerve fibers was found in the trigeminal root subjacent to the sites of microvascular compression in patients with TN; the pathological tissue changes included axonal loss, demyelination, and dysmyelination $(23,24)$.

Most recent studies have supported the view that interactions between astrocytes and neurons contribute to the synaptic plasticity mechanisms that help maintain chronic pain sensitization (25-27). Sensory axons span an abutment of peripheral and central nervous tissue along the trigeminal nerve root entry zones that anatomically connect the PNS with the CNS (28). The glial elements comprise the CNS-PNS interface of the TREZ (16). Notably, we found that GFAP-positive astrocytic processes showed a progressive extension from the proximal to the distal CNS-PNS boundary in the ipsilateral TREZ after CCT injury in our study. This implies that CCT may induce the activation of astrocytes in the CNS compartment of the TREZ in rats. This extension of astrocytic processes may reflect the functional alteration of peripheral tissue nociceptive pain signal transduction in the CCT animal model. There have been no previous reports concerning the extension of GFAP-positive astrocytic processes in the TREZ in TN animals. But we do believe that chronic compression of the trigemi- nal root alters the neural plasticity of TREZ. However, the mechanism for this extension is still unclear and may be an interesting direction for future TN research.

The MDH is critical for the processing of nociceptive primary afferent information from the orofacial regions (29-31), and it has a cytoarchitecture similar to the substantia gelatinosa of the spinal cord dorsal horn (32). Different animal models of peripheral nerve injury have indicated that nociceptive C-fibers participate in mechanical and thermal hyperalgesia and the development of neuropathic pain (33). It was found that there was a dramatic decrease in IB4 expression in the superficial dorsal horn following nerve injury (34). Peripheral nerve injury induces various changes in neurotransmitter and neuropeptide expression in the nervous system, such as decreases in the levels of SP and CGRP in the dorsal root ganglia and spinal cord corresponding to the injured nerve. As excitatory neurotransmitters, SP and CGRP localized in primary afferents play a key role in the nociceptive signaling for the initiation and maintenance of neuropathic pain. The decrease of SP and CGRP that we observed in MDH after CCT likely reflected changes in expression of these peptides in primary afferents, there was a significant relationship between allodynia and peptide expression levels (35).

\section{Conclusion}

In conclusion, our study has established a novel animal model for TN by chronic compression of the trigeminal nerve root in rats. Even though this model also has several limitations, such as CCT with a plastic filament only imitated mechanical compression injury of trigeminal root without mimicking complex vascular physiological features, it is still reasonable to believe that this animal model may be feasible and it will provide better opportunities for the further study of TN pathogenesis.

\section{Acknowledgments}

This work was supported by grants from the National Natural Science Foundation of China (Nos:30771133, 30971123 and 31010103909). The authors wish to thank the editorial board of Pain Physician for review and criticism in improving the manuscript. 


\section{References}

1. Sessle BJ. Peripheral and central mechanisms of orofacial pain and their clinical correlates. Minerva Anestesiol 2005; 71:117-136.

2. Obermann M, Yoon MS, Ese D, Maschke M, Kaube H, Diener HC, Katsarava Z. Impaired trigeminal nociceptive processing in patients with trigeminal neuralgia. Neurology 2007; 69:835841.

3. Robinson PP, Boissonade FM, Loescher AR, Smith KG, Yates JM, Elcock C, Bird EV, Davies SL, Smith PL, Vora AR. Peripheral mechanisms for the initiation of pain following trigeminal nerve injury. J Orofac Pain 2004; 18:287-292.

4. Vos BP, Strassman AM, Maciewicz RJ. Behavioral evidence of trigeminal neuropathic pain following chronic constriction injury to the rat's infraorbital nerve. J Neurosci 1994; 14:2708-2723.

5. Xu M, Aita M, Chavkin C. Partial infraorbital nerve ligation as a model of trigeminal nerve injury in the mouse: $\mathrm{Be}$ havioral, neural, and glial reactions. J Pain 2008; 9:1036-1048.

6. Khan A, Hargreaves KM. Animal models of orofacial pain. Methods Mol Biol 2010; 617:93-104.

7. Love S, Coakham HB. Trigeminal neuralgia: Pathology and pathogenesis. Brain 2001; 124:2347-2360.

8. Marinkovic S, Todorovic V, Gibo $\mathrm{H}$, Budec M, Drndarevic N, Pesic D, Jokovic $M$, Cetkovic $M$. The trigeminal vasculature pathology in patients with neuralgia. Headache 2007; 47:1334-1339.

9. Ramesh VG, Premkumar G. An anatomical study of the neurovascular relationships at the trigeminal root entry zone. Clin Neurosci 2009; 16:934-936.

10. Lovely TJ, Jannetta PJ. Microvascular decompression for trigeminal neuralgia. Surgical technique and long-term results. Neurosurg Clin N Am 1997; 8:11-29.

11. Kernisant M, Gear RW, Jasmin L, Vit JP, Ohara PT. Chronic constriction injury of the infraorbital nerve in the rat using modified syringe needle. J Neurosci Methods 2008; 172:43-47.

12. Milligan ED, Twining C, Chacur M, Biedenkapp J, O'Connor K, Poole S, Tracey K, Martin D, Maier SF, Watkins LR. Spinal glia and proinflammatory cytokines mediate mirror-image neuropathic pain in rats. J Neurosci 2003; 23:1026-1040.

13. Chaplan SR, Bach FW, Pogrel JW, Chung
JM, Yaksh TL. Quantitative assessment of tactile allodynia in the rat paw. J Neurosci Methods 1994; 53:55-63.

14. Imamura $\mathrm{Y}$, Kawamoto $\mathrm{H}$, Nakanishi $\mathrm{O}$. Characterization of heat-hyperalgesia in an experimental trigeminal neuropathy in rats. Exp Brain Res 1997; 116:97-103.

15. Mei XP, Wang W, Zhu C, Chen L, Zhang T, Xu LX, Wu SX, Li YQ. Combining ketamine with astrocytic inhibitor as a potential analgesic strategy for neuropathic pain ketamine, astrocytic inhibitor and pain. Mol Pain 2010; 6:50.

16. Toma JS, McPhail LT, Ramer MS. Comparative postnatal development of spinal, trigeminal and vagal sensory root entry zones. Int J Dev Neurosci 2006; 24:373-388.

17. Attal N, Filliatreau G, Perrot S, Jazat F, D Giamberardino L, Guilbaud G. Behavioural pain-related disorders and contribution of the saphenous nerve in crush and chronic constriction injury of the rat sciatic nerve. Pain 1994; 59:301-312.

18. Tal M, Eliav E. Abnormal discharge originates at the site of nerve injury in experimental constriction neuropathy $(\mathrm{CCl})$ in the rat. Pain 1996; 64:511-518.

19. Stuesse SL, Crisp T, McBurney DL, Schechter JB, Lovell JA, Cruce WL. Neuropathic pain in aged rats: Behavioral responses and astrocytic activation. Exp Brain Res 2001; 137:219-227.

20. Liang YC, Huang CC, Hsu KS. The synthetic cannabinoids attenuate allodynia and hyperalgesia in a rat model of trigeminal neuropathic pain. Neuropharmacology 2007; 53:169-177.

21. Vos BP, Hans G, Adriaensen H. Behavioral assessment of facial pain in rats: Face grooming patterns after painful and non-painful sensory disturbances in the territory of the rat's infraorbital nerve. Pain 1998; 76:173-178.

22. Hilton DA, Love S, Gradidge T, Coakham $H B$. Pathological findings associated with trigeminal neuralgia caused by vascular compression. Neurosurgery 1994; 35:299-303; discussion 303.

23. Devor M, Govrin-Lippmann R, Rappaport ZH. Mechanism of trigeminal neuralgia: An ultrastructural analysis of trigeminal root specimens obtained during microvascular decompression surgery. J Neurosurg 2002; 96:532-543.

24. Marinkovic S, Gibo H, Todorovic V, Antic B, Kovacevic D, Milisavljevic M, Cet- kovic M. Ultrastructure and immunohistochemistry of the trigeminal peripheral myelinated axons in patients with neuralgia. Clin Neurol Neurosurg 2009; 111:795-800.

25. Ren K. Emerging role of astroglia in pain hypersensitivity. Jpn Dent Sci Rev 2010; 46:86

26. Haydon PG, Carmignoto G. Astrocyte control of synaptic transmission and neurovascular coupling. Physiol Rev 2006; 86:1009-1031.

27. Ji RR, Kawasaki Y, Zhuang ZY, Wen YR, Decosterd I. Possible role of spinal astrocytes in maintaining chronic pain sensitization: Review of current evidence with focus on bFGF/JNK pathway. Neuron Glia Biol 2006; 2:259-269.

28. Aita M, Byers MR, Chavkin C, Xu M. Trigeminal injury causes kappa opioid-dependent allodynic, glial and immune cell responses in mice. Mol Pain 2010; $6: 8$.

29. Bereiter DA, Hirata H, Hu JW. Trigeminal subnucleus caudalis:beyond homologies with the spinal dorsal horn. Pain 2000; 88:221-224.

30. Sessle BJ. Acute and chronic craniofacial pain: Brainstem mechanisms of nociceptive transmission and neuroplasticity, and their clinical correlates. Crit Rev Oral Biol Med 2000; 11:57-91.

31. Davies AJ, Kim YH, Oh SB. Painful neuron-microglia interactions in the trigeminal sensory system. The Open Pain Journal 2010; 3:14-28.

32. Kerr FW. The fine structure of the subnucleus caudalis of the trigeminal nerve. Brain Res 1970; 23:129-145.

33. Basbaum Al, Bautista DM, Scherrer G, Julius D. Cellular and molecular mechanisms of pain. Cell 2009; 139:267-284.

34. Molander C, Wang HF, Rivero-Melian C, Grant G. Early decline and late restoration of spinal cord binding and transganglionic transport of isolectin $\mathrm{B}_{4}$ from Griffonia simplicifolia I after peripheral nerve transection or crush. Restor Neurol Neurosci 1996; 10:123-133.

35. Cameron AA, Cliffer KD, Dougherty PM, Garrison C), Willis WD, Carlton SM. Time course of degenerative and regenerative changes in the dorsal horn in a rat model of peripheral neuropathy. $]$ Comp Neurol 1997; 379:428-442. 